TITLE:

\title{
Strain relaxation effect by nanotexturing InGaN/GaN multiple quantum well
}

\section{$\operatorname{AUTHOR}(S)$ :}

Ramesh, V.; Kikuchi, Akihiko; Kishino, Katsumi; Funato, Mitsuru; Kawakami, Yoichi

\section{CITATION:}

Ramesh, V.... [et al]. Strain relaxation effect by nanotexturing InGaN/GaN multiple quantum well. JOURNAL OF APPLIED PHYSICS 2010, 107(11): 114303.

\section{ISSUE DATE:}

2010-06

URL:

http://hdl.handle.net/2433/147196

\section{RIGHT:}

Copyright 2010 American Institute of Physics. This article may be downloaded for personal use only. Any other use requires prior permission of the author and the American Institute of Physics. The following article appeared in JOURNAL OF APPLIED PHYSICS107, 114303 (2010) and may be found at 


\title{
Strain relaxation effect by nanotexturing InGaN/GaN multiple quantum well
}

\author{
V. Ramesh, ${ }^{1,2}$ A. Kikuchi, ${ }^{1,2}$ K. Kishino, ${ }^{1,2, a)}$ M. Funato, ${ }^{2,3}$ and Y. Kawakami ${ }^{2,3}$ \\ ${ }^{1}$ Department of Electrical and Electronics Engineering, Sophia University, Tokyo 102-8554, Japan and Nano- \\ technology Research Center, Sophia University, Tokyo 102-8554, Japan \\ ${ }^{2}$ CREST, JST, Saitama 332-0012, Japan \\ ${ }^{3}$ Department of Electronics Science and Engineering, Kyoto University, Kyoto 615-8510, Japan
}

(Received 31 December 2009; accepted 19 February 2010; published online 2 June 2010)

\begin{abstract}
The relaxation of lattice-mismatched strain by deep postetching was systematically investigated for InGaN/GaN multiple quantum wells (MQWs). A planar heterojunction wafer, which included an $\mathrm{In}_{0.21} \mathrm{Ga}_{0.79} \mathrm{~N}(3.2 \mathrm{~nm}) / \mathrm{GaN}(14.8 \mathrm{~nm}) \mathrm{MQW}$, was etched by inductively coupled plasma dry etching, to fabricate high-density nanopillar, nanostripe, and nanohole arrays. The etching depth was $570 \mathrm{~nm}$ for all nanostructures. The diameter of the nanopillars was varied from 50 to $300 \mathrm{~nm}$, then the mesa stripe width of the nanostripes and the diameter of the nanoholes were varied from $100 \mathrm{~nm}$ to $440 \mathrm{~nm}$ and $50 \mathrm{~nm}$ to $310 \mathrm{~nm}$, respectively. The effect of strain relaxation on various optical properties was investigated. For example, in an array of nanopillars with diameter $130 \mathrm{~nm}$ and interval $250 \mathrm{~nm}$, a large blueshift in the photoluminescence (PL) emission peak from $510 \mathrm{~nm}$ (as-grown) to $459 \mathrm{~nm}$ occurred at room temperature (RT). PL internal quantum efficiency (defined by the ratio of PL integral intensity at $300 \mathrm{~K}$ to that at $4.2 \mathrm{~K}$ ) was enhanced from $34 \%$ (as-grown) to $60 \%$, and the PL decay time at $4.2 \mathrm{~K}$ was reduced from $22 \mathrm{~ns}$ (as-grown) to $4.2 \mathrm{~ns}$. These results clearly indicate the reduction of lattice-mismatched strain by postetching, which enhanced strain reduction with decreasing nanopillar diameter down to a diameter of $130 \mathrm{~nm}$, where the strain reduction became saturated. The dependence of RT-PL decay time on nanopillar diameter was measured, and the surface nonradiative recombination velocity was estimated to be 5.8 $\times 10^{2} \mathrm{~cm} / \mathrm{s}$. This relatively slow rate indicates a little etching damage. (C) 2010 American Institute of Physics. [doi:10.1063/1.3369434]
\end{abstract}

\section{INTRODUCTION}

Nitride-based compound semiconductors have been recognized as a powerful direct band gap material for many applications such as high-temperature/power electronic devices $^{1}$ and light emitting diodes (LEDs) and laser diodes operating in the visible and UV ranges of the electromagnetic spectrum. ${ }^{2,3}$ Generally, InGaN/GaN multiple quantum wells (MQWs) are used as the active layers in nitride-based visible light emitters. ${ }^{4,5}$ The peak wavelength and efficiency of emission are strongly affected by the built-in internal electric field arising from the combination of spontaneous and piezoelectric polarization in the quantum wells $(\mathrm{QWs}){ }^{6}{ }^{6}$ the strong internal field tilts the band edge; thus, the quantumconfined stark effect induces a redshift in the emission spectrum. At the same time, low internal quantum efficiency (IQE) is caused by the reduced overlapping between the electron and hole wave functions. Under the current injection condition, however, the carrier-screening effect of the internal field restores the tilt of the band, bringing about a blueshift in the emission. The shift in the carrier-induced emission peak wavelength of the c-plane grown InGaN LED is therefore of concern for device applications.

Several methods have been proposed to reduce the strain-induced piezoelectric field in nitride-based QWs. The strain reduction has been performed by growing a p-InGaN layer on top of the active region ${ }^{7}$ or a $\mathrm{QW}$ with a composi-

${ }^{a)}$ Electronic mail: kishino@katsumi.ee.sophia.ac.jp. tionally graded barrier layer, ${ }^{8}$ or by inserting a prestrain layer prior to the growth of $\mathrm{QWs},{ }^{9,10}$ or by decreasing the QW thickness. ${ }^{11}$ An alternative approach to circumvent the generation of an internal field is to perform growth on nonpolar and semipolar planes. ${ }^{12}$ Several groups have recently reported the fabrication of $\mathrm{GaN}$ based QWs with a reduced strain-induced piezoelectric field using low-dimensional nanostructures that produced a blueshift in the PL wavelength. Two different approaches bottom-up and top-down, are utilized to fabricate $\mathrm{GaN}$ based nanostructures. For example, self-organized GaN nanocolumns ${ }^{13-15}$ have been fabricated by the bottom-up approach. Presence of a low internal field in InGaN QW nanocolumn was reported. ${ }^{15}$ There have also recently been many reports on adapting the topdown approach to prepare nanostructures by a combination of electron-beam lithography (EBL) and inductively coupled plasma (ICP)/RIE etching, ${ }^{16,17}$ or by using nanoimprint technology, ${ }^{18}$ as well as to fabricate nanopillars by direct etching using a focused ion beam (FIB) (Refs. 19 and 20) and nanoporous masking materials. ${ }^{21,22}$ Although strain relaxation and the consequent blueshift in the emission wavelength were reported in these studies, the slight increase in PL intensity, reduction in PL decay time, and the strain relaxation effect of postetching a nanostructure with an InGaN QW observed in these studies are not systematically understood yet. It was also pointed out that the fabrication process can seriously damage the sample surface and lead to a significant decrease in luminescence of the MQW, even after optimized annealing. ${ }^{16}$ Recently, we have reported the opti- 
cal properties of $\mathrm{InGaN} / \mathrm{GaN}$ top-down nanopillars fabricated by chemically assisted ion beam etching, ${ }^{23}$ in which cylindrical shapes of nanostructure with diameters of $2 \mu \mathrm{m}$, $1 \mu \mathrm{m}$, or $500 \mathrm{~nm}$ were prepared. This experiment proved that partial strain relaxation occurs at the free side wall but strain remains in the middle of the pillar structures because still a bit large diameter of nanopillar does not completely relax the strain. Nevertheless the strain relaxation leads to an enhanced radiative recombination rate.

In this article, the strain relaxation in an InGaN/GaN MQW was investigated for various styles of top-down nanostructure, i.e., nanopillar, nanostripe, and nanohole structures with a dramatic downsizing the nanostructures into length scale less than $100 \mathrm{~nm}$. The nanostructures were fabricated by ICP dry etching using a thick $\mathrm{SiO}_{2}$ mask to avoid the formation of etching defects. The strain relaxation effect was examined by performing microphotoluminescence ( $\mu$-PL) and time-resolved PL (TR-PL) measurements to estimate the nonradiative surface recombination velocity. Furthermore, the size dependence of the strain relaxation of the nanostructures was investigated by systematically varying the sizes of the nanopillar, nanostripe, and nanohole structures.

\section{EXPERIMENT}

Five-period MQWs composed of 3.2-nm-thick $\mathrm{In}_{\mathrm{x}} \mathrm{Ga}_{1-\mathrm{x}} \mathrm{N}$ wells and 14.8-nm-thick $\mathrm{GaN}$ barriers capped by a 15-nm-thick GaN layers were grown by metal organic chemical vapor deposition on a 3 - $\mu$ m-thick Si-doped GaN layer prepared on a (0001) sapphire substrate. The thickness and average indium (In) composition were estimated from the high-resolution $\mathrm{x}$-ray diffraction rocking curve of the (0002) plane reflection. The average In composition was $21 \%$ in each QW.

The InGaN/GaN MQW was etched through a nanopattern mask. In the process, a 100 -nm-thick $\mathrm{SiO}_{2}$ film was first deposited by sputtering on the as-grown wafer to reduce the etching damage and increase the selectivity of GaN etching. Then a 5-nm-thick Ti conducting film was deposited by evaporation to prevent the charging during EB exposure; finally, nanostructures were prepared through EBL followed by ICP etching. Nanostructures with various stripe [different interval (L) and mesa width (W)], hole, and pillar [different $\mathrm{L}$ and diameter $(\Phi)]$ configurations were prepared with a depth of about $570 \mathrm{~nm}$. Because the QWs were located at a depth of $105 \mathrm{~nm}$ from the top surface, $570 \mathrm{~nm}$ etching is sufficient to reach the underlying GaN:Si layer. The nanopillars and nanoholes were prepared in a triangular lattice arrangement. After the etching process, EBL resist and $\mathrm{SiO}_{2}$

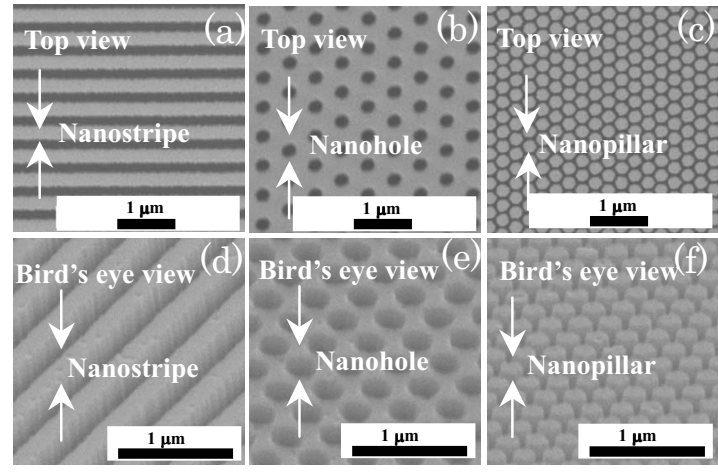

FIG. 1. Top and bird's-eye-view SEM images of (a) and (d) nanostripe (L $=400 \mathrm{~nm}, \mathrm{~W}=210 \mathrm{~nm}), \quad(\mathrm{b})$ and (e) nanohole $(\mathrm{L}=500 \mathrm{~nm}, \Phi$ $=200 \mathrm{~nm})$, and (c) and (f) nanopillar $(\mathrm{L}=250 \mathrm{~nm}, \Phi=200 \mathrm{~nm})$ samples.

were removed with a suitable etchant, then the dimensions and density of the nanostructures were measured by scanning electron microscope (SEM). The emission properties were observed at room temperature $(\mathrm{RT}=300 \mathrm{~K})$ and a low temperature $(4.2 \mathrm{~K})$ by $\mu$-PL measurements. Here, the samples were excited by the $325 \mathrm{~nm}$ line of a He-Cd laser through a $(40 \times)$ objective lens with an excitation power of $1.8 \mathrm{~mW}$; the optical power was measured immediately above the sample surface. The excitation power density was kept constant for all measurements. The luminescence was collected through the objective lens and transmitted through an optical fiber to a spectrometer with an electrically cooled chargecoupled device detector.

\section{RESULTS AND DISCUSSION}

Typical SEM images of five-well InGaN/GaN MQWs with nanopillars, nanostripes, and nanoholes are shown in Fig. 1. The nanopillar and nanohole densities were maintained in the range from $6.25 \times 10^{8}$ to $2.5 \times 10^{9} \mathrm{~cm}^{-2}$, when the dimensions of the nanopillars, nanostripes, and the nanoholes were varied over a wide range as shown in Table I. The bird's eye view SEM images show the fabrication of a smooth side surface, and the estimated etching depth was about $570 \mathrm{~nm}$. All nanostructures were narrower at the top than at the bottom, and the slope angle of the sidewalls was $87.5^{\circ}$.

Figure 2 shows a comparison of the RT-PL spectrum of the as-grown planar MQW and nanopillar structures, where nanopillar diameter was varied from $200 \mathrm{~nm}$ to $50 \mathrm{~nm}$ under a constant period, $\mathrm{L}=250 \mathrm{~nm}$. The PL peak wavelength of the as-grown MQW sample was $510 \mathrm{~nm}$, where as for the nanopillars, a blueshift was observed with decreasing pillar diameter $(\Phi)$; the PL wavelengths of the samples with $\Phi$

TABLE I. Intervals and dimensions of the nanopillars, nanostripes, and nanoholes.

\begin{tabular}{|c|c|c|c|c|c|}
\hline \multicolumn{2}{|c|}{ Stripe pattern } & \multicolumn{2}{|c|}{ Hole pattern } & \multicolumn{2}{|c|}{ Pillar pattern } \\
\hline $\begin{array}{l}\text { Period, L } \\
\quad(\mathrm{nm})\end{array}$ & $\begin{array}{l}\text { Stripe width, W } \\
(\mathrm{nm})\end{array}$ & $\begin{array}{l}\text { Period, L } \\
\quad(\mathrm{nm})\end{array}$ & $\begin{array}{l}\text { Diameter, } \Phi \\
(\mathrm{nm})\end{array}$ & $\begin{array}{l}\text { Period, L } \\
\quad(\mathrm{nm})\end{array}$ & $\begin{array}{l}\text { Diameter, } \Phi \\
\quad(\mathrm{nm})\end{array}$ \\
\hline 250 & 70-200 & 250 & $50-155$ & 250 & 50-200 \\
\hline 300 & $70-260$ & 300 & $55-210$ & 300 & $70-220$ \\
\hline 400 & $190-360$ & 400 & 70-290 & 400 & $120-300$ \\
\hline
\end{tabular}




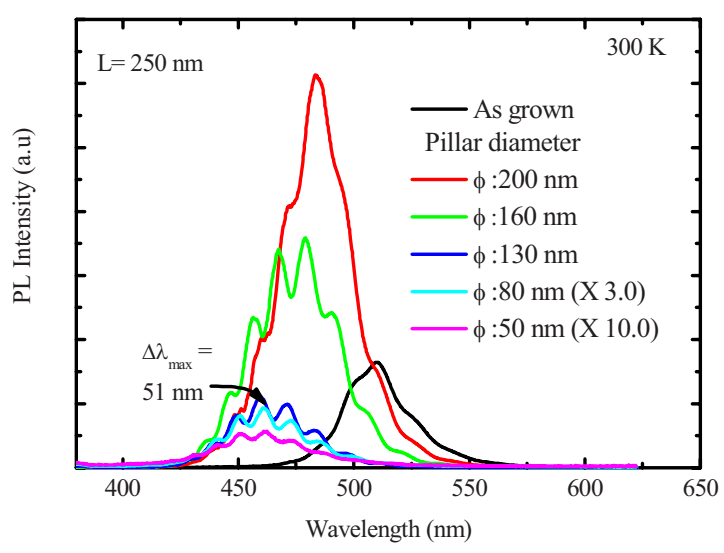

FIG. 2. (Color online) Comparison of PL spectra of as-grown planar sample and InGaN/GaN MQW nanopillar with $\Phi=200 \mathrm{~nm}, 160,130,80$, and 50 $\mathrm{nm}$ measured at RT.

$=200,160,130,80$, and $50 \mathrm{~nm}$ were $482 \mathrm{~nm}, 475 \mathrm{~nm}, 459$ $\mathrm{nm}, 459 \mathrm{~nm}$, and $460 \mathrm{~nm}$, respectively. Interestingly, when $\Phi$ was below $130 \mathrm{~nm}$, no further blueshift occurred. Thus, the shortest peak wavelength attained was $459 \mathrm{~nm}$, giving a maximum blueshift $(\Delta \lambda)$ of $51 \mathrm{~nm}$, but a marked decrease in the PL intensity occurred with decreasing volume of the active region. Note that the blueshift of $51 \mathrm{~nm}$ is much higher than the values reported for randomly arranged FIB-etched nanopillars ${ }^{19}$ and ICP-etched nanopillars/stripes. ${ }^{16,18,24}$ This blueshift can be attributed to two factors such as: the strain relaxation ${ }^{25,26}$ and the quantum size effect ${ }^{24,27}$ of the MQW. However, the blueshift based on the quantum size effect is negligibly small for nanopillars with large diameters of above $15 \mathrm{~nm}^{24}$ Thus, strain relaxation caused the blueshift, ${ }^{16,17,23,28}$ proving that strain relaxation occurs for nanopillars with diameters of $1 \mu \mathrm{m}$ or less. Because samples in this experiment possessed a smaller diameter of 50-200 $\mathrm{nm}$, they should undergo greater strain relaxation in the MQW region.

Similar strain relaxation was observed for the nanostripe and nanohole structures, but the blueshift was small, probably because the occupation ratio of the etched sidewall, on which the strain relaxation preferentially occurs, was small. To take differences in configuration of the nanostructures into account, the spatial occupation factor of the sidewall is: $\mathrm{V}_{\text {soc }}$ (sidewall length/unetched area) was defined. Figure 3 shows the blueshift $(\Delta \lambda)$ as a function $V_{\text {soc }} . \Delta \lambda$ increased with $\mathrm{V}_{\text {soc }}$ until $\mathrm{V}_{\text {soc }}$ value reached $5.0 \times 10^{-3} \mathrm{~nm}^{-1}$ (typically, nanopillars with $\Phi=130 \mathrm{~nm}$ and $\mathrm{L}=250 \mathrm{~nm}$ ) but above that, no change was observed, owing to the saturation of the strain relaxation effect. Strain relaxation only occurs at the area of the etched sidewall, thus, it is independent of the configuration of the nanostructures; this experiment clarified that the amount of strain relaxation is determined by $\mathrm{V}_{\mathrm{soc}}$, independent of the type of nanostructures. Figure 4 shows PL intensity at RT as a function of $\mathrm{V}_{\text {soc }}$; note that the PL intensity increased sharply up to a $V_{\text {soc }}$ of $1.3 \times 10^{-3} \mathrm{~nm}^{-1}$ and then decreased abruptly. This phenomenon is explained as follows. Below a $\mathrm{V}_{\text {soc }}$ of $1.3 \times 10^{-3} \mathrm{~nm}^{-1}$ an increase in $\mathrm{V}_{\text {soc }}$ enhances strain relaxation, which improves the emission efficiency of the InGaN-MQW, thus increasing the light intensity. However, when $\mathrm{V}_{\text {soc }}$ increases above this value the

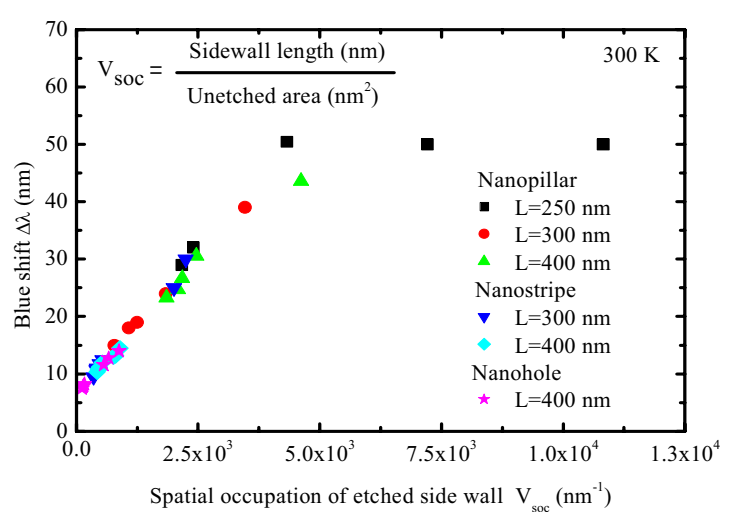

FIG. 3. (Color online) Measured blueshift $(\Delta \lambda)$ of the nanostructures with relative to as-grown MQW as a function of $\mathrm{V}_{\text {soc }}$ (spatial occupation of etched sidewall).

volume of the active region decreases substantially, reducing the emission intensity. Below a $\mathrm{V}_{\text {soc }}$ of $5.0 \times 10^{-3} \mathrm{~nm}^{-1}$, the strain relaxation effect probably increases the intensity but it is overcome by the volume reduction effect but above this value the strain relaxation effect is saturated, and, therefore, the intensity decreased to below that of the original as-grown planar sample owing to the volume reduction in the active region. Thus, the PL intensity was maximized at $\mathrm{V}_{\mathrm{soc}}=1.3$ $\times 10^{-3} \mathrm{~nm}^{-1}$, at which for the nanopillar structure with $\mathrm{L}$ $=300 \mathrm{~nm}$ and $\Phi=200 \mathrm{~nm}$, the PL intensity was enhanced by a factor of about 8 compared with that of the as-grown planar sample. Note that all data, including that for nanostripe and nanoholes follow the same trend, as shown in Fig. 4 , indicating that the configuration of the nanostructures did not provide the difference in the PL intensity, which was determined uniquely by the spatial occupation of the sidewall.

The improvement in the PL intensity is understood as follows. The strain relaxation restores the tilted band to the flat band by increasing the overlapping between the electron and hole wave functions, which increases the light oscillation strength and enhances the radiative recombination rate. At the same time, nanotexturing effect of the structures can enhance light extraction efficiency from InGaN QW. However, as discussed in Ref. 23, no enhancement in light extraction is

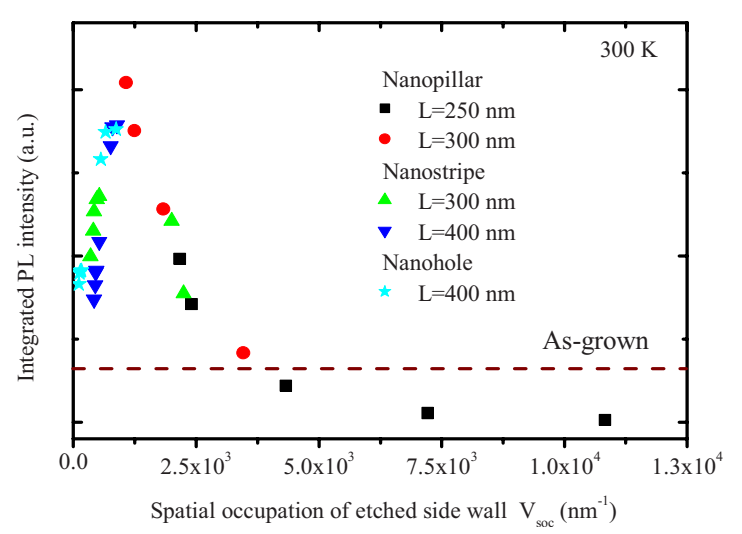

FIG. 4. (Color online) PL intensity of the nanostructures as a function of $\mathrm{V}_{\text {soc }}$ (spatial occupation of etched sidewall). 


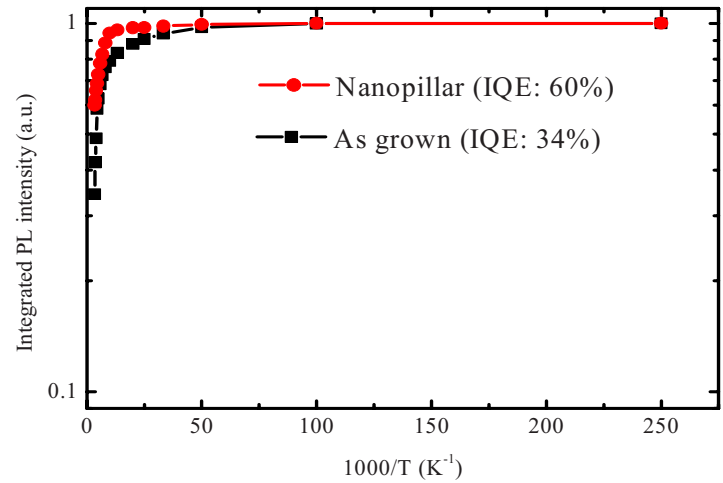

FIG. 5. (Color online) Arrhenius plots of the PL integrated intensities of as-grown MQW film and nanopillar sample ( $\mathrm{L}=400 \mathrm{~nm}$, and $\Phi=250 \mathrm{~nm})$.

projected when the InGaN QW is placed closely to the surface, for example, in nanopillar with a very thin capping layer (15 $\mathrm{nm}$ in thickness). ${ }^{23}$

The temperature dependences of the PL integrated intensity $I(T)$ for the as-grown planar sample and nanopillar sample with $\mathrm{L}=400 \mathrm{~nm}$ and $\Phi=250 \mathrm{~nm}$ were evaluated in the temperature range from 300 to $4.2 \mathrm{~K}$ using an Oxford Instruments cryostat system. The result is shown in Fig. 5, where $I(T)$ was normalized to unity at the lowest temperature. $I(T)$ for the as-grown planar MQW and nanopillar samples was almost constant at low temperatures and then sharply decreased with increasing temperature above $100 \mathrm{~K}$ and $175 \mathrm{~K}$, respectively. This tendency indicates that the activation temperature of the nonradiative process was higher for the nanopillar sample, suggesting a higher IQE at RT. PL-IQE is defined as the ratio of RT-PL integrated intensity to that at the low temperature $(4.2 \mathrm{~K})$, at which nonradiative recombination processes are assumed to be completely quenched. ${ }^{29,30}$ The PL-IQE was evaluated by varying the incident power density of the excitation laser source from $1 \mu \mathrm{W} / \mathrm{cm}^{2}$ to $1.0 \mathrm{~mW} / \mathrm{cm}^{2}$ and was maximized at $700 \mu \mathrm{W} / \mathrm{cm}^{2}$; the maximum PL-IQE at RT was about $34 \%$ for the as-grown sample, and $60 \%$ for the nanopillar sample with $\mathrm{L}=400 \mathrm{~nm}$ and $\Phi=250 \mathrm{~nm}$. This enhancement in PLIQE was caused by the strain relaxation, and induced an increase in the recombination probability of carriers.

Furthermore, the samples were evaluated by TR-PL measurements at $4.2 \mathrm{~K}$. Figure 6 shows the TR spectra of the as-grown MQW and nanopillar samples $(\mathrm{L}=250 \mathrm{~nm}$, and $\Phi=130 \mathrm{~nm}$ ). Both PL time decay curves were fit to the following stretched exponential expression: ${ }^{31}$

$$
I(t)=A_{1} e\left(\frac{-t}{\tau_{1}}\right)+A_{2} e\left(\frac{-t}{\tau_{2}}\right)^{\beta}
$$

where $I(t)$ is PL intensity as a function of time $t, \tau_{1}$ and $\tau_{2}$ are recombination time constants, and $\beta$ is a scaling parameter related to the dimensionality of the localizing centers (here, $\beta=0.7$ ). ${ }^{8,31,32}$

The fitting gave PL decay times of the as-grown planar MQW and the nanopillar samples of $22 \mathrm{~ns}$ and $4.2 \mathrm{~ns}$, respectively. The dependence of PL decay time on nanopillar diameter was investigated, and it was found that the decay time is reduced with decreasing diameter and becomes satu-

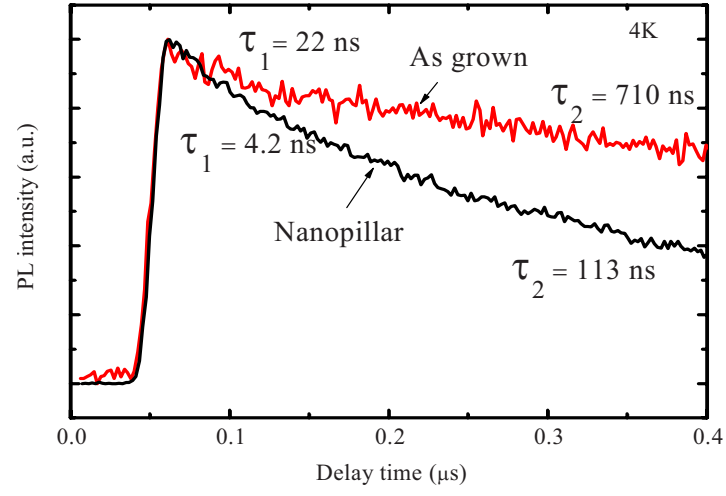

FIG. 6. (Color online) TR-PL decay time for the as-grown and nanopillar $(\mathrm{L}=250 \mathrm{~nm}$, and $\Phi=130 \mathrm{~nm}$ ) samples measured at $4 \mathrm{~K}$. The decay time was derived by fitting a stretched exponential expression. $\tau_{1}$ and $\tau_{2}$ are decay components.

rated at $4.2 \mathrm{~ns}$ when $\Phi=130 \mathrm{~nm}$. From a numerical simulation of the strain distribution of an InGaN quantum disk embedded in a GaN nanopillar in the radial direction, the strain relaxation of the quantum disk occurs at the side-wall region at a depth of 10-20 nm from the side surface. Such a strain distribution has previously been reported for GaN/ AlGaN nanocolumns ${ }^{33}$ and InGaN/GaN nanopillars. ${ }^{23}$ Note that faster radiative recombination occurs in the strainrelaxed region, and the diffusion length of carriers in InGaN MQWs was reported to be between 100 and $200 \mathrm{~nm} .{ }^{34}$ Fast recombination at the sidewall region produces a radial carrier distribution, which induces the driving force of carrier diffusion from the nanopillar center to the side facet. Thus, from the experimental results it can be understood that, when the nanopillar diameter was less than $130 \mathrm{~nm}$, almost all the carriers were recombined with faster recombination in the strain-relaxed sidewall with assistance as a result of carrier diffusion. Actually, more complicated mechanism comes into play in the emission because the carrier diffusion should be analyzed under the radial energy distribution taking both the strain relaxation induced energy-change and the surface energy pinning model into account. But the experimental results of Figs. 3 and 4 support that the carrier diffuses completely to the sidewall when the nanostructure sizes are sufficiently small. But a precise analysis of emission mechanism is remained in future. In addition to that, we notice that the $\mu$-PL spectra of the nanopillar structures with diameter less then $130 \mathrm{~nm}$ were completely separated from that of the as-grown sample; thus, the emission at the center strain region was sufficiently suppressed. In fact, when the diameter decreased to below $130 \mathrm{~nm}$, no further blueshift in the PL spectrum occurred (Fig. 3).

In this section, the surface recombination process in the nanopatterned MQW is discussed. Room temperature TR-PL measurement was carried out for nanopillar structures with different $\mathrm{L}$ ( 250 to $400 \mathrm{~nm}$ ) and $\Phi(50$ to $300 \mathrm{~nm}$ ) to estimate the decay time $\left(\tau_{\mathrm{PL}}\right)$ using the stretched exponential fitting. The decay process consisted of radiative and nonradiative recombination; thus, the RT-PL decay time $\left(\tau_{\mathrm{PL}}\right)$ is expressed as $^{35}$ 


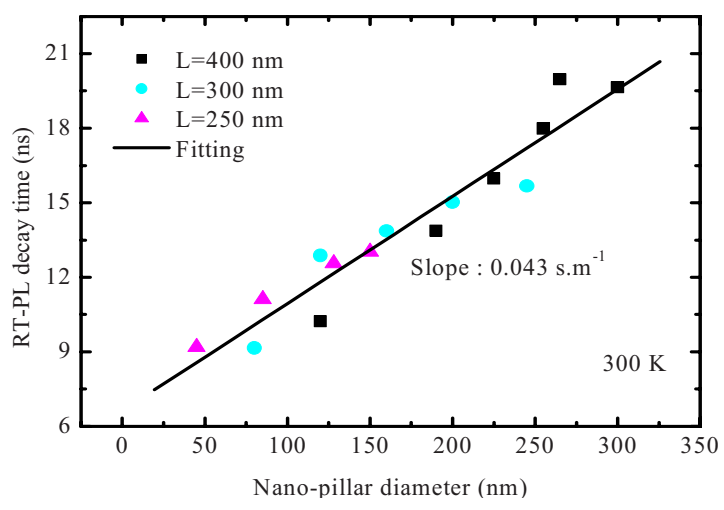

FIG. 7. (Color online) Plot of nonradiative decay time vs the nanopillar diameter. From the slope of the fitting line, nonradiative surface recombination velocity $\mathrm{V}_{\mathrm{s}}$ of $\left(5.79 \times 10^{2} \mathrm{~cm} / \mathrm{s}\right)$ was calculated.

$$
\frac{1}{\tau_{(\mathrm{PL})}}=\frac{1}{\tau_{(\mathrm{rad})}}+\frac{1}{\tau_{(\text {nonrad })}}
$$

where $\tau_{(\mathrm{rad})}$ and $\tau_{\text {(non-rad) }}$ are the radiative and nonradiative decay times, respectively. Also IQE is expressed in terms of $\tau_{(\mathrm{rad})}$ and $\tau_{\text {(nonrad) }}$ as

$$
\mathrm{IQE}=\frac{\tau_{(\mathrm{nonrad})}}{\tau_{(\mathrm{rad})}+\tau_{(\mathrm{nonrad})}} .
$$

Using Eqs. (2) and (3) and the measured $\tau_{(\mathrm{PL})}$ and IQE values, the radiative and nonradiative decay times were separately evaluated. Figure 7 shows the estimated $\tau_{\text {(nonrad) }}$ as a function of nanopillar diameter; $\tau_{\text {(nonrad) }}$ is linearly dependent on the diameter, which clearly indicates that the surface recombination governs the decay process. It was also confirmed that nanostructures with different configurations, i.e., nanopillar, nanostripe, and nanohole, had the same recombination lifetime for the same sidewall spatial occupation.

For nanopillars with a cylindrical cross section, the nonradiative surface recombination lifetime $\tau_{\text {(nonrad) }}$ can be written as $d /\left(4 V_{s}\right)$, where $d$ is the pillar diameter and $V_{s}$ is the surface recombination velocity. This formula matches the expected surface recombination time for a filament with a square cross section, ${ }^{26,36}$ but it differs from that for a planar confinement structure $\left(\tau_{(\text {nonrad })}=\mathrm{T} /\left(2 \mathrm{~V}_{\mathrm{s}}\right)\right)$ in which a planar active layer of thickness $\mathrm{T}$ is bound by only two surfaces/interfaces. ${ }^{37}$ Assuming that $\left(\tau_{(\text {nonrad })}=\mathrm{T} /\left(4 \mathrm{~V}_{\mathrm{s}}\right)\right)$ applies to our InGaN MQW nanopillars with cylindrical cross sections, the slope in Fig. 7 gives $1 /\left(4 \mathrm{~V}_{\mathrm{s}}\right)$. Thus, from the linear fitting of the data, the RT nonradiative surface recombination velocity is estimated to be $V_{\mathrm{s}}=5.8 \times 10^{2} \mathrm{~cm} / \mathrm{s}$. Note that this value is about one order smaller than that for Si-doped GaN nanowires; ${ }^{26}$ it was as a factor of 20 smaller than the reported value for undoped $\mathrm{GaN}$ epilayers, ${ }^{32}$ and two orders of magnitude smaller than the reported value for GaAs $\left(V_{s}=1 \times 10^{6} \mathrm{~cm} / \mathrm{s}\right){ }^{38}$ And it was a factor of 2 smaller than the value reported for an InGaN-based photonic crystal structure. ${ }^{39}$ Therefore, the low surface recombination velocity and enhanced emission efficiency make the nanopillar material a good candidate for nanoscale light-emittingdevice applications.

\section{CONCLUSIONS}

High-density $\left(6.25 \times 10^{8}\right.$ to $\left.2.5 \times 10^{9} \mathrm{~cm}^{-2}\right)$ top-down nanohole, nanostripe, and nanopillar InGaN/GaN MQW structures were fabricated by ICP dry etching, where the lateral dimensions of the nanostructures were varied over a wide range with a constant height of $570 \mathrm{~nm}$. The RT-PL emission wavelength of the nanopillars with interval $250 \mathrm{~nm}$ and diameter less then $130 \mathrm{~nm}$ was evaluated to be $459 \mathrm{~nm}$, indicating a blueshift of $51 \mathrm{~nm}$ relative to emission wavelength of an as-grown planar MQW sample. The blueshift is caused by the strain relaxation effect; the strain relaxation improved the emission intensity by a factor of 8 and the PL-IQE value from $34 \%$ to $60 \%$, causing LT-PL decay time, corresponding to the radiative lifetime, to be shortened from $20 \mathrm{~ns}$ to $4.2 \mathrm{~ns}$. This improved PL property was obtained as a result of suppressed internal field, which induced an increase in the overlapping between electron and hole wave functions, in addition to the improved light extraction obtained by nanotexturing effect. It was also clarified that the strain relaxation effect occurred independent of the type of nanostructure, in which the amount of relaxation was determined only by the role of spatial occupation of the etched sidewall. The nonradiative surface recombination velocity on the etched sidewall of the InGaN MQW was evaluated to be $\mathrm{V}_{\mathrm{s}}=5.8 \times 10^{2} \mathrm{~cm} / \mathrm{s}$. This value is extremely low compared with previously reported data. From these experiments, it can therefore be concluded that the top-down approach to fabricating nanostructures is applicable for the fabrication of InGaN based nanolight-emitting devices.

\section{ACKNOWLEDGMENTS}

This study was supported in part by a Grant-in-Aid for Scientific Research on Priority Areas in Japan (A) Grant No. 18069010 and (B) Grant No. 21310087 from MEXT-JP.

${ }^{1}$ S. Nakamura, Science 281, 956 (1998).

${ }^{2}$ S. Nakamura and G. Fasol, The Blue Laser Diode (Springer, Berlin, 2000). ${ }^{3}$ M. Asif Khan, M. Shatalov, H. P. Maruska, H. M. Wang, and E. Kuokstis, Jpn. J. Appl. Phys., Part 1 44, 7191 (2005).

${ }^{4}$ T. Mukai, M. Yamada, and S. Nakamura, Jpn. J. Appl. Phys., Part 1 38, 3976 (1999).

${ }^{5}$ S. Nakamura, M. Senoh, S. Nagahama, N. Iwasa, T. Yamada, T. Matsushita, H. Kiyoku, and Y. Sugimoto, Jpn. J. Appl. Phys., Part 2 35, L74 (1996).

${ }^{6}$ T. Takeuchi, S. Sota, M. Katsuragawa, M. Komori, H. Takeuchi, H. Amano, and I. Akasaki, Jpn. J. Appl. Phys., Part 2 36, L382 (1997).

${ }^{7}$ J. H. Ryou, W. Lee, J. Limb, D. Yoo, J. P. Liu, R. D. Dupuis, Z. H. Wu, A. M. Fischer, and F. A. Ponce, Appl. Phys. Lett. 92, 101113 (2008).

${ }^{8}$ T. Onuma, Y. Uchinuma, E. K. Suh, H. J. Lee, T. Sota, and S. F. Chichibu, Jpn. J. Appl. Phys., Part 2 42, L1369 (2003).

${ }^{9}$ C. F. Huang, C. Y. Chen, C. F. Lu, and C. C. Yang, Appl. Phys. Lett. 91, 051121 (2007)

${ }^{10}$ W. Y. Shiao, C. F. Huang, T. Y. Tang, J. J. Huang, Y. C. Lu, C. Y. Chen, Y. S. Chen, and C. C. Yang, J. Appl. Phys. 101, 113503 (2007).

${ }^{11}$ J. Bai, T. Wang, and S. Sakai, J. Appl. Phys. 88, 4729 (2000).

${ }^{12}$ T. Takeuchi, H. Amano, and I. Akasaki, Jpn. J. Appl. Phys., Part 1 39, 413 (2000).

${ }^{13}$ M. Yoshizawa, A. Kikuchi, N. Fujita, K. Kushi, H. Sasamoto, and K. Kishino, Jpn. J. Appl. Phys., Part 2 36, L459 (1997).

${ }^{14}$ A. Kikuchi, M. Kawai, M. Tada, and K. Kishino, Jpn. J. Appl. Phys., Part 2 43, L1524 (2004).

${ }^{15}$ Y. Kawakami, S. Suzuki, A. Kaneta, M. Funamoto, A. Kikuchi, and K. Kishino, Appl. Phys. Lett. 89, 163124 (2006).

${ }^{16}$ S. Keller, C. Schaake, N. A. Fichtenbaum, C. J. Neufeld, Y. Wu, K. Mc- 
Groddy, A. David, S. P. DenBaars, C. Weisbuch, J. S. Speck, and U. K. Mishra, J. Appl. Phys. 100, 054314 (2006).

${ }^{17}$ T. H. Hsueh, H. W. Huang, C. C. Kao, Y. H. Chang, M. Chia, O. Yang, H. C. Kuo, and S. C. Wang, Jpn. J. Appl. Phys., Part 1 44, 2661 (2005).

${ }^{18}$ H. Ono, Y. Ono, K. Kashara, J. Mizuno, and S. Shoji, Jpn. J. Appl. Phys. 47, 933 (2008).

${ }^{19}$ S. E. Wu, T. H. Hsueh, C. P. Liu, J. K. Sheu, W. C. Lai, and S. J. Chang, Jpn. J. Appl. Phys. 47, 3130 (2008).

${ }^{20}$ S. E. Wu and C. P. Liu, Nanotechnology 16, 2507 (2005).

${ }^{21}$ C. Y. Wang, L. Y. Chen, C. P. Chen, Y. W. Cheng, M. Y. Ke, M. Y. Hsieh, H. M. Wu, L. H. Peng, and J. J. Huang, Opt. Express 16, 10549 (2008).

${ }^{22}$ H. W. Huang, J. T. Chu, T. H. Hsueh, M. C. Ou-Yang, H. C. Kuo, and S C. Wang, J. Vac. Sci. Technol. B 24, 1909 (2006).

${ }^{23}$ Y. Kawakami, A. Kaneta, L. Su, Y. Zhu, K. Okamoto, M. Funato, A Kikuchi, and K. Kishino, J. Appl. Phys. 107, 023522 (2010).

${ }^{24}$ H. S. Chen, D. M. Yeh, Y. C. Lu, C. Y. Chen, C. F. Huang, T. Y. Tang, C. C. Yang, C. S. Wu, and C. D. Chen, Nanotechnology 17, 1454 (2006).

${ }^{25}$ H. J. Chang, Y. P. Hsieh, T. T. Chen, Y. F. Chena, and C. T. Liang, Opt. Express 15, 9357 (2007).

${ }^{26}$ J. B. Schlager, K. A. Bertness, P. T. Blanchard, L. H. Robins, A. Roshko, and N. A. Sanford, J. Appl. Phys. 103, 124309 (2008).

${ }^{27}$ R. W. Martin, P. R. Edwards, H. S. Kim, K. S. Kim, T. Kim, I. M. Watson, M. D. Dawson, Y. Cho, T. Sands, and N. W. Cheung, Appl. Phys. Lett. 79, 3029 (2001).

${ }^{28}$ L. Dai, B. Zhang, J. Y. Lin, and H. X. Jiang, J. Appl. Phys. 89, 4951
(2001).

${ }^{29}$ T. Akasaka, H. Gotoh, H. Nakano, and T. Makimoto, Appl. Phys. Lett. 86, 191902 (2005).

${ }^{30}$ D. M. Graham, P. Dawson, M. J. Godfrey, M. J. Kappers, P. M. F. J. Costa, M. E. Vickers, R. Datta, C. J. Humphreys, and E. J. Thrush, Phys. Status Solidi C 3, 1970 (2006).

${ }^{31}$ Y. J. Sun, O. Brandt, S. Cronenberg, S. Dhar, H. T. Grahn, and K. H. Ploog, Phys. Rev. B 67, 041306 (2003).

${ }^{32}$ R. Aleksiejūnas, M. Sudzius, T. Malinauskas, J. Vaitkus, K. Jarasiunas, and S. Sakai, Appl. Phys. Lett. 83, 1157 (2003).

${ }^{33}$ J. Ristić, C. Rivera, E. Calleja, and S. Fernández-Garrido, Phys. Rev. B 72, 085330 (2005).

${ }^{34}$ K. Kumakura, T. Makimoto, N. Kobayashi, T. Hashizume, T. Fukui, and H. Hasegawa, J. Cryst. Growth 298, 787 (2007).

${ }^{35}$ Y. Narukawa, Y. Kawakami, S. Fujita, and S. Nakamura, Phys. Rev. B 59, 10283 (1999).

${ }^{36}$ W. Shockly, Electrons and Holes in Semiconductors (Van Nostrand, New York, 1950).

${ }^{37}$ R. K. Ahrenkiel, Minority Carriers in III-V Semiconductors: Physics and Applications (Academic, Boston, 1993), Vol. 39, p. 39.

${ }^{38}$ J. Lloyd-Hughes, S. K. E. Merchant, L. Fu, H. H. Tan, C. Jagadish, E. Castro-Camus, and M. B. Johnston, Appl. Phys. Lett. 89, 232102 (2006).

${ }^{39}$ H. Kitagawa, T. Suto, M. Fujita, Y. Tanaka, T. Asano, and S. Noda, Appl. Phys. Express 1, 032004 (2008). 